\title{
Laurence-Moon syndrome
}

INSERM

\section{Source}

INSERM. (1999). Orphanet: an online rare disease and orphan drug data base. Laurence-

Moon syndrome. ORPHA:2377

Laurence-Moon syndrome (LMS) is a very rare genetic multisystemic disorder characterized by pituitary dysfunction, ataxia, peripheral neuropathy, spastic paraplegia, and chorioretinal dystrophy. 